\title{
BOREL GAMES AND THE BAIRE PROPERTY \\ BY
}

\author{
KENNETH SCHILLING AND ROBERT VAUGHT
}

\begin{abstract}
The Borel game operations are a natural generalization of the operation (A). It is shown that these operations preserve the property of Baire in all topological spaces. Applications are given to invariant descriptive set theory and the model theory of infinitary logic.
\end{abstract}

Introduction. It is a classical theorem that the operation (A) preserves the Baire property in an arbitrary topological space. There are two proofs-e.g., in Kuratowski's Topology [6]. One $(\S 11, \mathrm{VII})$ is valid in general; while the other $(\$ 19, \mathrm{I}, 4)$ works only if some countability assumption is made for the space (e.g., for a separable metric, but not for an arbitrary metric space). ${ }^{1}$

Corresponding to each Borel subset of $\mathscr{P}(\omega)$ is an operation $G_{L}$ which can be construed as a generalization of the operation (A). It is called a Borel game operation. D. A. Martin [7] established the fundamental fact that all Borel games are determinate. Using Martin's result, R. Solovay showed that the Borel game operations preserve the Baire property in all spaces which are Polish (separable, complete metrizable). In this paper, we show (Theorem A) that the result holds in arbitrary topological spaces. At the same time, using a recently discovered method of Kechris [5], we shall (in Theorem B) extend to all spaces a result of Burgess, which locates in a useful way the position of the outcome set when $G_{L}$ is applied 'mod meager', in terms of the input sets 'mod meager'.

We also will show that these results have a $\kappa$-version in which games are played on an arbitrary infinite cardinal $\kappa$, and meager, Baire property, etc., are replaced by their usual $\kappa$-versions.

In $\S 1$, a detailed statement of the results is given.

1. Detailed statement of results. Let $R$ be any nonempty set and let $r$ (usually subscripted) range over $R$. Suppose $Q$ is a subset of $R^{\omega}$ (the set of all functions on the set $\omega$ of natural numbers to $R$ ). We write

$$
\forall r_{0} \exists r_{1} \forall r_{2} \exists r_{3} \cdots\left(r_{0}, r_{1}, r_{2}, r_{3}, \ldots\right) \in Q
$$

to mean that player II has a winning strategy in the (infinite) game in which I plays $r_{0}$, II plays $r_{1}$, I plays $r_{2}$, etc., and II wins if $r=\left(r_{0}, r_{1}, r_{2}, \ldots\right) \in Q$. (A winning strategy for player II is a function $F$ on the $\operatorname{set} \operatorname{Sq}(R)$ of all nonempty finite

Received by the editors November 15, 1982

1980 Mathematics Subject Classification. Primary 04A15, 54H05; Secondary 03C75.

'In fact, the first such (topological) theorem discovered was a version of the first proof simplified by a countability assumption! See [6] for a history involving Lusin, Sierpinski, Nikodym, Szpilrajn-Marczewski, Banach and Selivanowski. 
sequences of elements of $R$ to $R$ such that, for any $\left(r_{0}, r_{2}, r_{4}, \ldots\right)$, if $r_{1}=F\left(\left(r_{0}\right)\right)$, $r_{3}=F\left(\left(r_{0}, r_{2}\right)\right)$, etc., then $\left(r_{0}, r_{1}, r_{2}, r_{3}, \ldots\right) \in Q$.) We write

$$
\exists r_{0} \forall r_{1} \exists r_{2} \forall r_{3} \cdots\left(r_{0}, r_{1}, r_{2}, r_{3}, \ldots\right) \notin Q
$$

if player I has a winning strategy in the above game. This assertion is called the dual of the assertion (*). It is not difficult to show, using the axiom of choice, that there exist $R$ and $Q$ for which $(*)$ and $(* *)$ both fail. The game is then said to be indeterminate.

It is easy to see (and we shall often use) that, e.g., (*) holds only if $\forall r_{0}\left(\exists r_{1} \forall r_{2} \exists r_{3}\right.$ $\cdots r \in Q$ ) holds.

Give $R^{\omega}$ the product topology based on the discrete topology for $R$. The game above is called a Borel game if $Q$ is Borel in $R^{\omega}$. Martin's theorem [7] states that if the game is Borel, then it is determinate. One easily verifies that $Q \subseteq R^{\omega}$ is Borel if and only if $Q=\left\{r \in R^{\omega}:\left\{n:\left(r_{0}, \ldots, r_{n}\right) \in W\right\} \in L\right\}$ for some $W \subseteq \mathrm{Sq}(r)$ and some $L$ Borel in $\mathscr{P}(\omega)$. ( $\mathscr{P}(B)$ is the power set of $B$ and has the usual topology from its twin $2^{B}$.)

Let $K$ be a fixed nonempty set, whose members are denoted by $k$ (subscripted). (Usually below, $K$ will be $\omega$.) Suppose $L \subseteq \mathscr{P}(\omega)$ and $X$ is a nonempty set. If $A=\left(A_{s}: s \in \operatorname{Sq}(K)\right)$ is any system of subsets of $X$, then $G_{L}^{X, K}(A)$ (the $(K, L)$-game operation applied to the system $A$ ) is the set of all $x \in X$ such that

$$
\forall k_{0} \exists k_{1} \forall k_{2} \exists k_{3} \cdots\left(\left\{n: x \in A_{k_{0} k_{1} \cdots k_{n}}\right\} \in L\right) .
$$

If $K=\omega$, we omit ' $K$ ' and speak of the "game operation $G_{L}^{X}$ "; often $X$ is also omitted. If $L$ is Borel in $\mathcal{P}(\omega)$, the above operation is, by definition, a Borel $K$-game operation. The operation (A) is roughly the case where $K=\omega, L$ is closed, and II is the only player. Note that if $x$ is fixed, the game above is indeed a Borel game.

Henceforth in this paper, $X$ will be an arbitrary topological space, and $\Re$ will be a weak basis for $X$ (that is, a family of nonempty open sets such that for any nonempty open $\theta$, there is a $U \in \mathscr{B}$ such that $U \subseteq \vartheta$. One can, for example, take $\mathscr{B}$ to be the family of all nonempty open sets). The letters $U$ and $V$, usually subscripted, will range over $\mathscr{B}$. If $A, B \subseteq X$, write $A \leqslant B$ to mean that $A-B$ is meager. $A \equiv B$ means that $A \leqslant B$ and $B \leqslant A$. $A$ has the Baire property if for some open set $\mathcal{O}$, $A \equiv \mathcal{O} . X$ is a Baire space if no nonempty open set is meager. We will often use without mention the fact (see, e.g., [6]) that if $A \subseteq \theta$ and $\theta$ is open, then $A$ is meager in (the sense of the subspace) $\theta$ if and only if $A$ is meager.

We can now state the generalized Solovay and Burgess theorems (to be proved in §).

Theorem. Let $L$ be a Borel subset of $\mathscr{P}(\omega)$. Suppose for each $s \in \operatorname{Sq}(\omega), A_{s}$ has the Baire property in the topological space $X$. Then

(A) (generalized Solovay) $G=G_{L}^{X . \omega}(A)$ has the Baire property; and

(B) (generalized Burgess) if $X$ is a Baire space, then for any open set $\theta, \theta \leqslant G$ if and only if

(1) $\forall U_{0} \subseteq \vartheta \forall k_{0} \exists U_{1} \subseteq U_{0} \exists k_{1} \forall U_{2} \subseteq U_{1} \forall k_{2} \cdots\left(\left\{n: \exists m U_{m} \leqslant A_{k_{0} \cdots k_{n}}\right\} \in L\right)$. 
Solovay and Burgess [1] proved (A) and (B), respectively, assuming that $X$ is Polish (more or less). These proofs as given certainly seem not to work in spaces with no type of countability condition. Theorem (B), for the case when $G_{L}^{X, \omega}$ is the operation (A), was obtained only recently by the second author [13]. (See [4] for an independent related result.)

More recently Kechris [5] gave a beautiful new proof of both the Solovay and the Burgess results, one of whose features is that the two are proved together. (In fact, everything follows from one direction of Burgess's theorem.) Kechris dealt with spaces with a countable basis which can be represented in a particular way (see §4). We show in (A) and (B) that Burgess's result, as well as Solovay's, extends to arbitrary topological spaces. Our proof follows the method of Kechris.

The proofs of (A) and (B) and related matters occupy $\$ \S 2-5$. In $\$ 6$ we consider an arbitrary infinite cardinal $\kappa$. In any space $X, A \subseteq X$ is called $\kappa$-meager if it is the union of at most $\kappa$ (ordinary) nowhere dense sets. The notions $A \leqslant{ }_{\kappa} B, A \equiv_{\kappa} B$, and $\kappa$-Baire property are defined in exact analogy to the case $\kappa=\omega$. (E.g., $A$ has the

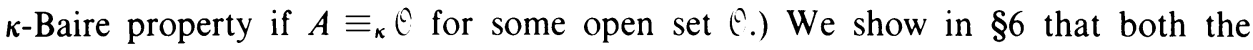
Solovay and Burgess theorems generalize to the $\kappa$-notions; that is, (A) and (B) hold if $\mathrm{Sq}(\omega)$ is replaced by $\mathrm{Sq}(\kappa)$, Baire property by $\kappa$-Baire property, $\leqslant$ by $\leqslant_{\kappa}$, and $G_{L}^{X, \omega}$ by $G_{L}^{X, \kappa}$.

Burgess applied his work to the ${ }^{*}$-transform of [13], which can take place in a topological action, and, in particular, in the "logic action". to obtain new results of the form of Lopez-Escobar's "invariant Borel $=L_{\omega_{1}, \omega}$ ". In the "bad' $X$ to which we extend Burgess's result, this procedure fails in a sense because $X$ has no countable basis. However, applying our $\kappa$-results in this way works perfectly for $L_{\kappa+\kappa}$. The results obtained are new even when $L$ is at the lowest level, i.e., closed. They fill a gap mentioned explicitly in [13] where no $\kappa$-analogue was found for the earlier results there about the operation (A). These matters are considered in $\S 7$.

Solovay's original results applied not only to the Borel game operations, but also to a wider class of Boolean operations, the so-called absolutely $\Delta_{2}^{\prime}$ operations. (Solovay never published these results, though they do appear with proof in [2].) These results were slightly modified and generalized by the second author in unpublished work announced at a meeting in Wrockaw in 1977. Since then the first author has obtained a full generalization of Solovay's theorem (and the present Theorem (A)): roughly, absolutely $\Delta_{2}^{1}$ operations preserve the Baire property in all topological spaces. (See [11].)

Solovay's theorem, and the second author's generalization, apply also when "Baire property and meager" are replaced by "measurable and measure zero" for suitable measure spaces, and even to an arbitrary $\sigma$-field and ideal, provided the induced Boolean algebra satisfies appropriate countability conditions. More recently, the first author has succeeded in adapting the arguments herein to cover these other cases. All these matters will be considered in a paper to appear soon.

2. More background. Let $I$ be any set and $S \subseteq \mathcal{P}(I)$. Given any set $X, S$ determines an $I$-ary operation $\vartheta_{S}^{X}$ over $\mathscr{P}(X)$, where

$$
\vartheta_{S}^{X}\left(\left(A_{i}: i \in I\right)\right)=\left\{x:\left\{i: x \in A_{i}\right\} \in S\right\} \text {. }
$$


(Reference to $X$ is often omitted.) Operations so obtainable are called Boolean. Note that, given $L \subseteq \mathcal{P}(\omega)$ and a fixed $K$, the game operation $G_{L}^{X, K}$ is Boolean; in fact $G_{L}^{X, K}=\mathcal{O}_{S}^{X}$ where $I=\operatorname{Sq}(K)$, and $W \in S$ if and only if

$$
\forall k_{0} \exists k_{1} \forall k_{2} \cdots\left(\left\{n:\left(k_{0}, k_{1}, \ldots, k_{n}\right) \in W\right\} \in L\right) .
$$

The following fact is well known and easily proved.

2.1. If $\vartheta$ is an I-ary Boolean operation for $X$ where $I$ is countable, and $A_{i} \equiv B_{i}$ for each $i \in I$, then $\Theta(A) \equiv \Theta(B)$.

From 2.1 one sees at once that the Solovay Theorem (A) and the Burgess Theorem (B) reduce to the case where all of the sets $A_{s}$ are open.

Suppose $\leqslant$ is a relation on the set $R$ such that for any $r \in R$ there is an $s \in R$ such that $s \leqslant r$. Strictly speaking the (already used) notation

$$
\forall r_{0} \exists r_{1} \leqslant r_{0} \forall r_{2} \leqslant r_{1} \cdots r \in P
$$

needs clarification. It is easy to see that the following two possible interpretations of (2) are equivalent.

$$
\forall r_{0} \exists r_{1} \forall r_{2} \cdots\left((\forall n \neq 0)\left(r_{2 n} \leqslant r_{2 n-1}\right) \rightarrow \forall n\left(r_{2 n+1} \leqslant r_{2 n} \text { and } r \in P\right)\right)
$$

and

$\left(2^{\prime \prime}\right)$ there exists a function $F$ such that

(i) $F$ is defined on the set of all sequences $\left(r_{0}, r_{2}, \ldots, r_{2 n}\right)$ such that, for $i=1,2, \ldots, n, F\left(\left(r_{0}, r_{2}, \ldots, r_{2 i-2}\right)\right)$ is defined and $r_{2 i} \leqslant F\left(\left(r_{0}, \ldots, r_{2 i-2}\right)\right)$;

(ii) $F\left(\left(r_{0}, r_{2}, \ldots, r_{2 n}\right)\right) \leqslant r_{2 n}$; and

(iii) if $r=\left(r_{0}, r_{1}, r_{2}, \ldots\right)$ is a play according to $F$, i.e., $r_{0} \geqslant r_{1} \geqslant r_{2} \geqslant r_{3} \geqslant \cdots$, and $r_{1}=F\left(\left(r_{0}\right)\right), r_{3}=F\left(\left(r_{0}, r_{2}\right)\right)$, etc., then $r \in P$.

Either may be taken as the definition of (2). Using $\left(2^{\prime}\right)$ one sees that, if $P$ is Borel in $R^{\omega}$, then the game referred to by (2) is a Borel game. In fact, let $\Pi_{\xi}^{0}, 1 \leqslant \xi<\omega_{1}$, denote the standard Borel classifications as, for example, in [13]. Then using ( $\left.2^{\prime}\right)$ one can see that if $P$ is a $\Pi_{\xi}^{0}$ set with $\xi \geqslant 2$, the game referred to by (2) is a $\Pi_{\xi}^{0}$ game. (This also holds for $\xi=1$; see [13, p. 276].)

3. The generalized Banach-Mazur theorem. The Banach-Mazur theorem expresses the condition " $B$ is comeager" in terms of a game.

Theorem 3.1 (BANACH-Mazur). Let $B \subseteq X$. Then

$$
\forall U_{0} \exists U_{1} \subseteq U_{0} \forall U_{2} \subseteq U_{1} \exists U_{3} \subseteq U_{2} \cdots \bigcap_{h \in \omega} U_{n} \subseteq B
$$

if and only if $B$ is comeager.

This is proved in [9]. A simpler proof of the main (left-to-right) implication for the case when $X$ has a countable basis is implicit in [5].

Let $P \subseteq X \times \omega^{\omega}$. (We sometimes write, e.g., $P x k$ or $P(x, k)$ for $(x, k) \in P$.) Following a modified Kechris-style approach, we shall later derive both main theorems (A) and (B) from the following Theorem (C). 
If

$$
\forall U_{0} \forall k_{0} \exists U_{1} \subseteq U_{0} \exists k_{1} \forall U_{2} \subseteq U_{1} \forall k_{2} \cdots\left(\bigcap_{m \in \omega} U_{m} \subseteq\{x \in X: P x k\}\right),
$$

then $\left\{x \in X: \forall k_{0} \exists k_{1} \forall k_{2} \cdots P x k\right\}$ is comeager.

(C) can be regarded as a generalization or strengthening of the main implication of the Banach-Mazur theorem, which is obtained by taking $P x k$ if $x \in B$. For certain $P$ (the main ones used below) (C) is just one direction of Burgess's theorem, and so (C) was proved by Burgess for these $P$ if $X$ is Polish. Kechris [5] stated and proved (C) for arbitrary $P$ for his special, countably based $X$.

There are two quite different proofs of (C). One, the longer of the two, must repeat and complicate the proof of the Banach-Mazur theorem for arbitrary $X$. This proof also works for the $\kappa$-version of (C) and will be given in $\$ 6$. The other, which works only for $\omega$, is apparently shorter as it succeeds in using the Banach-Mazur theorem. In fact we shall show that (C) follows immediately from a "game theorem" which is a generalization to the uncountable of a theorem of Kechris [5].

4. The game theorem. Let $(C, \leqslant)$ be a partially ordered set, and let subscripted $p$ range over elements of $C$. Let $Z$ be the set of $p \in C^{\omega}$ such that $p_{0} \geqslant p_{1} \geqslant p_{2} \geqslant \cdots$. Define the equivalence relation $E$ on $Z$ by $p E q$ if for each $m$ the sequence $q$ is eventually $\leqslant p_{m}$, and vice versa. Let $X=X_{C}=Z / E$, the set of all equivalence classes $p / E$ for $p \in Z$.

Aside. $X_{C}$ can be given a topology so that the sets $\left\{p / E\right.$ : for some $\left.n, p_{n} \leqslant r\right\}$ for $r \in C$ form a basis. These spaces $X_{C}$ for $C$ countable are just the spaces which Kechris [5] considers, e.g., in connection with Theorems (A) and (B). For $C$ countable, he obtains the following theorem, which we now prove in general.

Theorem 4.1 (The Game Theorem). Let $Q \subseteq X_{C} \times \omega^{\omega}$. If

$$
\forall p_{0} \forall k_{0} \exists p_{1} \leqslant p_{0} \exists k_{1} \forall p_{2} \leqslant p, \forall k_{1} \cdots Q(p / E, k),
$$

then

$$
\forall p_{0} \exists p_{1} \leqslant p_{0} \forall p_{2} \leqslant p_{1} \cdots\left(\forall k_{0} \exists k_{1} \forall k_{2} \cdots Q(p / E, k)\right) .
$$

Proof. Let $F$ be a winning strategy for $(*)$. One can easily find a bijection $s: \omega \rightarrow \mathrm{Sq}(\omega)$ such that $i \leqslant j$ whenever $s_{i} \subseteq s_{j}$. ( $s_{i}$ is an initial piece of $s_{j}$.) If $t \in \operatorname{Sq}(\omega)$, put $\#(t)=s^{-1}(t)$. Put $v(i, j)=\#\left(s_{i} \uparrow\{0,1, \ldots, j\}\right)$.

We describe a winning strategy for II in the $p_{i}$-game in $(* *)$. Suppose I has played $\left(q_{0}, q_{1}, \ldots, q_{k}\right) . k$ determines $s_{k}=\left(m_{0}, m_{1}, \ldots, m_{n}\right)$. For $i \leqslant n, s_{v(k, i)}=$ $\left(m_{0}, m_{1}, \ldots, m_{i}\right)$ and clearly $0 \leqslant v(k, 0)<v(k, 1)<\cdots<v(k, n)=k$. We may consider $z=\left(q_{v(k, 0)}, m_{0}, \ldots, q_{v(k, n)}, m_{n}\right)$ as a list of player I's first $n+1$ moves of the game (*). Applying our strategy to $z$ we get $F(z)=(r, e)$. We now have II play $r$. On the side he 'remembers' $e$ by defining an auxiliary function $u$; he puts $u\left(q_{0}, \ldots, q_{k}\right)=e$.

Notice that if $n<n^{\prime}, \#\left(\left(m_{0}, \ldots, m_{n}\right)\right)=k, \#\left(\left(m_{0}, \ldots, m_{n^{\prime}}\right)\right)=k^{\prime}$ and $i \leqslant n$, then both sequences have the same reduction to $\{0, \ldots, i\}$, so $v(k, i)=v\left(k^{\prime}, i\right)$. Informally, II is simulating many games of type $(*)$ during one play of $(* *)$. 
Suppose now that $p=\left(q_{0}, r_{0}, q_{1}, r_{1}, \ldots\right)$ is a full play of the $p$-part of $(* *)$ following our strategy. We must construct a strategy showing that

$$
\forall i_{0} \exists j_{0} \forall i_{1} \exists j_{1} \cdots Q\left(p / E,\left(i_{0}, j_{0}, \ldots\right)\right) .
$$

Suppose I has played to date (in $(* * *))\left(i_{0}, \ldots, i_{n}\right)$. Let $k=\#\left(i_{0}, \ldots, i_{n}\right)$ so that $\left(i_{0}, \ldots, i_{n}\right)=s_{k}$. In playing $(* *)$ above after I played $q_{0}, \ldots, q_{k}$, we had 'in mind' $\left(i_{0}, \ldots, i_{n}\right)$. So II now plays (in $\left.(* * *)\right) m\left(\left(q_{0}, \ldots, q_{k}\right)\right)$.

Let $\left(i_{0}, j_{0}, i_{1}, j_{1}, \ldots\right)$ be a full play of $(* * *)$ by this strategy. For any $n$, let $k(n)=\#\left(\left(i_{0}, \ldots, i_{n}\right)\right)$. Put $q_{n}^{\prime}=q_{k(n)}$ and $r_{n}^{\prime}=r_{k(n)}$. Then clearly $\left(q_{0}^{\prime}, i_{0}, r_{0}^{\prime}, j_{0}, q_{1}^{\prime}, i_{1}, r_{1}^{\prime}, j_{1}, \ldots\right)$ is a (correctly descending) play of $(*)$ according to the strategy $F$. Hence $Q\left(\left(q_{0}^{\prime}, r_{0}^{\prime}, q_{1}^{\prime}, r_{1}^{\prime}, \ldots\right) / E,\left(i_{0}, j_{0}, i_{1}, j_{1}, \ldots\right)\right)$ holds. But

$$
\left(q_{0}^{\prime}, r_{0}^{\prime}, q_{1}^{\prime}, r_{1}^{\prime}, \ldots\right)=\left(q_{k(0)}, r_{k(0)}, q_{k(1)}, r_{k(1)}, \ldots\right),
$$

which is $E$-equivalent to $\left(q_{0}, r_{0}, q_{1}, r_{1}, \ldots\right)$. Hence $Q\left(\left(q_{0}, r_{0}, q_{1}, r_{1}, \ldots\right) / E\right.$, $\left.\left(i_{0}, j_{0} i_{1}, j_{1}, \ldots\right)\right)$, as desired.

We conclude this section with two useful lemmas.

Lemma 4.2. Let $K$ be a set and $Q \subseteq X_{C} \times K^{\omega}$. Let $F$ be a winning strategy for

$$
\forall p_{0} \forall k_{0} \exists p_{1} \leqslant p_{0} \exists k_{1} \cdots Q(p / E, k) .
$$

Suppose further that $G$ is another strategy for (!) such that, for any play $z=$ $\left(p_{0}, k_{0}, p_{2}, k_{2}, \ldots, p_{2 n}, k_{2 n}\right)$ of $(!)$ if $G(z)=(q, m)$ and $F(z)=\left(q^{\prime}, m^{\prime}\right)$, then $q \leqslant q^{\prime}$ and $m=m^{\prime}$. Then $G$ is also a winning strategy for (!).

Proof. Suppose that $\left(p_{0}, k_{0}, p_{1}, k_{1}, p_{2}, k_{2}, \ldots\right)$ is a play of (!) in which II has followed the strategy $G$. We must show that $Q(p / E, k)$ holds.

Consider the sequence $\left(p_{0}, k_{0}, p_{1}^{\prime}, k_{1}, p_{2}, k_{2}, p_{3}^{\prime}, k_{3}, \ldots\right)$, where, for all $n, p_{2 n+1}^{\prime}$ $=F\left(\left(p_{0}, k_{0}, p_{2}, k_{2}, \ldots, p_{2 n}, k_{2 n}\right)\right)$, that is, the result of playing the strategy $F$ against I's previous moves. Then $p_{2 n+1} \leqslant p_{2 n+1}^{\prime}$ by assumption. Clearly $p_{2 n+2} \leqslant$ $p_{2 n+1}$, and so $p_{2 n+2} \leqslant p_{2 n+1}^{\prime}$. This shows that $\left(p_{0}, k_{0}, p_{1}^{\prime}, k_{1}, \ldots\right)$ is actually a play of the game (!). Since it is a play according to the winning strategy $F$, we have $Q\left(\left(p_{0}, p_{1}^{\prime}, p_{2}, p_{3}^{\prime}, \ldots\right), k\right)$. But clearly the sequence $\left(p_{0}, p_{1}^{\prime}, p_{2}, p_{3}^{\prime}, \ldots\right)$ is $E$-equivalent to $p$, so we have $Q(p / E, k)$, as desired.

For any $p, q \in C, p$ and $q$ are said to be incompatible if there is no $r \in C$ such that $r \leqslant p$ and $r \leqslant q$. We say that $p$ 'resolves' $q$ just in case either $p \leqslant q$ or $p$ is incompatible with $q$. Note that if $p^{\prime} \leqslant p$ and $p$ resolves $q$, then so does $p^{\prime}$, and that for all $p$ and $q$, there is a $p^{\prime} \leqslant p$ that resolves $q$.

As a corollary of 4.2 we have

Proposition 4.3 (Resolving Lemma). Let $K$ be any set, $Q \subseteq X_{C} \times K^{\omega}$. Let $\left(a_{s}: s \in \mathrm{Sq}(K)\right)$ be a system of elements of $C$. Then if

$$
\forall p_{0} \forall k_{0} \exists p_{1} \exists k_{1} \cdots Q(p / E, k),
$$

there is a winning strategy $G$ for (!) such that, for any play $\left(p_{0}, k_{0}, p_{1}, k_{1}, \ldots\right)$ of (!) played according to $G$, for every odd number $n, p_{n}$ resolves $a_{k_{0} \cdots k_{n-1}}$ and $a_{k_{0} \cdots k_{n}}$ (So, in particular, for all $n, p_{n+1}$ resolves $a_{k_{0} \cdots k_{n}}$.) 
Proof. Let $F$ be a winning strategy for (!). We describe the strategy $G$.

Suppose play to date has gone $\left(p_{0}, k_{0}, \ldots, p_{n-1}, k_{n-1}\right), n$ odd (so it is II's move). Say $F$ tells II to play $\left(p_{n}, k_{n}\right)$. As remarked above, there is $p_{n}^{\prime} \leqslant p_{n}$ such that $p_{n}^{\prime}$ resolves $a_{k_{0} \cdots k_{n-1}}$, and $p_{n}^{\prime \prime} \leqslant p_{n}^{\prime}$ such that $p^{\prime \prime}$ resolves $a_{k_{0} \cdots k_{n}}$ (and $a_{k_{0} \cdots k_{n-1}}$ ). II plays $p_{n}^{\prime \prime}$.

It is clear that this strategy $G$ satisfies the hypotheses of 4.2 , and so is a winning strategy for (!). It is also clear that in any play according to $G$, the desired resolving takes place. This completes the proof.

By the same proof we get $4.3^{\prime}$, which is the same as 4.3 except that (!) is replaced by

$$
\exists p_{0} \exists k_{0} \forall p, \forall k_{1} \cdots Q(p / E, k)
$$

and the work 'odd' is everywhere replaced by 'even'.

5. On $G_{L}(A)$. We now apply the results of $\S 4$ to the topological space $X$. Theorem (C) is almost immediate from 4.1 .

Theorem (C). If

$$
\forall U_{0} \forall k_{0} \exists U_{1} \subseteq U_{0} \exists k_{1} \forall U_{2} \subseteq U_{1} \forall k_{2} \cdots\left(\bigcap_{m \in \omega} U_{m} \subseteq\{x \in X: P x k\}\right),
$$

then $\left\{x \in X: \forall k_{0} \exists k_{1} \forall k_{2} \cdots P x k\right\}$ is comeager.

Proof. Consider $(C, \subseteq)$ where $C$ is the weak basis 9 . Obviously we can let $Q(U / E, k)$ if and only if $\cap_{m \in \omega} U_{m} \subseteq\{x: P x k\}$. Hence by applying 4.1, we have

$$
\forall U_{0} \exists U_{1} \subseteq U_{0} \cdots\left(\forall k_{0} \exists k_{1} \cdots\left(\forall x \in \bigcap_{m \in \omega} U_{m}\right) P x k\right) .
$$

This condition clearly implies

$$
\forall U_{0} \exists U_{1} \subseteq U_{0} \cdots\left(\bigcap_{m \in \omega} U_{m} \subseteq\left\{x: \forall k_{0} \exists k_{1} \cdots P x k\right\}\right) .
$$

(The first one says there is a single strategy for all $x$, the second a strategy for each $x$.) By the Banach-Mazur theorem (3.1) it follows that $\left\{x: \forall k_{0} \exists k_{1} \cdots P x k\right\}$ is comeager, as desired.

We can now obtain a variant of $(C)$ which is very close to one direction of (B). For the remainder of this section we fix $L$ ( $L$ is not yet assumed to be Borel) and sets $A_{s} \subseteq X$ (for $s \in \mathrm{Sq}(\omega)$ ). We assume the $A_{s}$ are open; by 2.1 this does not change (A) and (B).

Theorem 5.1 (COROLlary of (C)). If

$$
\forall U_{0} \forall k_{0} \exists U_{1} \subseteq U_{0} \exists k_{1} \cdots\left\{n: \exists m U_{m} \subseteq A_{k_{0}} \cdots k_{n}\right\} \subseteq L,
$$

then

$$
G=G_{L}(A)=\left\{x: \forall k_{0} \exists k_{1} \cdots\left\{n: x \in A_{k_{0} \cdots k_{n}}\right\} \in L\right\}
$$

is comeager. (Note that $\left(\mathrm{l}^{\prime}\right)$ is similar to $(1)$ in $\S 1$ with $\subseteq$ in place of $\leqslant$. ) 
Proof. Let $P(x, k)$ if and only if $\left\{n: x \in A_{k_{0} \cdots k_{n}}\right\} \in L$. The conclusion of 5.1 will follow from (C) if we can obtain the hypothesis of (C), namely (3) above.

Play $\left(1^{\prime}\right)$ by the strategy guaranteed by 4.3 , with $(C, \leqslant)=(\Re, \subseteq), K=\omega$, $Q(U / E, k)$ if and only if $\left\{n: \exists m U_{m} \subseteq A_{k_{0} \cdots k_{n}}\right\} \in L$, and $a_{s}=A_{s}$. Suppose $\left(U_{0}, k_{0}, U_{1}, k_{1}, \ldots\right)$ is a play in this way, and $x \in \cap_{m \in \omega} U_{m}$. Then we know that $U_{n+1}$ resolves $A_{k_{0} \cdots k_{n}}$, and $\left\{n: \exists m U_{m} \subseteq A_{k_{0} \cdots k_{n}}\right\} \in L$. We want to show Pxk, that is $\left\{n: x \in A_{k_{0} \cdots k_{n}}\right\} \in L$, so it is clearly enough to show $x \in A_{k_{0} \cdots k_{n}}$ if and only if $\exists m U_{m} \subseteq A_{k_{0} \cdots k_{n}}$. From right to left this is obvious. For the converse, suppose there is no $m$ such that $U_{m} \subseteq A_{k_{0}} \cdots k_{n}$. Then, since $U_{n+1}$ resolves $A_{k_{0} \cdots k_{n}}, U_{n+1}$ and $A_{k_{0} \cdots k_{n}}$ are incompatible in $(\mathscr{G}, \subseteq)$. But since $\mathscr{G}$ is a weak basis, this implies that $U_{n+1} \cap$ $A_{k_{0} \cdots k_{n}}=\varnothing$. Thus $x \notin A_{k_{0} \cdots k_{n}}$. This completes the proof.

Notice that

5.2. If $L$ is Borel, the game in either (1) or ( $\left.1^{\prime}\right)$ is a Borel game (and, hence, by Martin's theorem, determinate).

Proof. The claim is, say, that the set of all $z=\left(U_{0}, k_{0}, U_{1}, k_{1}, \ldots\right)$ such that $\left\{n: \exists m U_{m} \subseteq A_{k_{0} \cdots k_{n}}\right\} \in L$ is Borel in $\mathscr{G}^{\omega} \times \omega^{\omega}$. Clearly $L$ being Borel, this reduces to showing $\left\{\left(U_{0}, k_{0}, \ldots\right): \exists m U_{m} \subseteq A_{k_{0} \cdots k_{n}}\right\}$ is Borel, and this in turn to the obvious fact that each $\left\{\left(U_{0}, k_{0}, \ldots\right): U_{m} \subseteq A_{k_{0} \cdots k_{n}}\right\}$ is Borel.

REMARK. It seems that an essential improvement by Kechris of Burgess's method was to apply Martin's theorem to the game ( $\left.1^{\prime}\right)$ rather than games $\forall k_{0} \exists k_{1} \cdots$. Notice that for us in game (1), Martin's result may be needed for games played on an uncountable set (even if $\kappa=\omega$ ).

We want to consider the dual assertion, for $(1)$ or $\left(1^{\prime}\right)$,

$$
\exists U_{0} \exists k_{0} \forall U_{1} \subseteq U_{0} \forall k_{1} \cdots\left\{n: \exists m U_{m} \subseteq A_{k_{0} \cdots k_{n}}\right\} \notin L .
$$

First, fix any $U_{0}$ and $k_{0}$. Then (as is easily checked)

$$
\forall U_{1} \subseteq U_{0} \forall k_{1} \exists U_{2} \subseteq U_{1} \exists k_{2} \cdots\left\{n: \exists m U_{m} \subseteq A_{k_{0} \cdots k_{n}}\right\} \notin L
$$

is equivalent to

$$
\forall U_{0}^{\prime} \subseteq U_{0} \forall k_{0}^{\prime} \exists U_{1}^{\prime} \subseteq U_{0}^{\prime} \exists k_{1}^{\prime} \cdots\left\{n: \exists m U_{m}^{\prime} \subseteq B_{k_{0}^{\prime} \cdots k_{n}^{\prime}}\right\} \in \sim L
$$

where $B_{k_{0}^{\prime} \cdots k_{p}^{\prime}}=A_{k_{0} k_{0}^{\prime} \cdots k_{p-1}^{\prime}} \cap U_{0}$. (Loosely speaking $U_{i}^{\prime}=U_{i+1}$ and $k_{1}^{\prime}=k_{i+1}$.) $\left(5^{\prime}\right)$ is exactly of the form $\left(1^{\prime}\right)$ (with $U_{0}$ for $X$ and $B_{s}$ for $A_{s}$ ). We now easily get

THEOREM 5.3. If the game $\left(1^{\prime}\right)$ is determinate (in particular if $L$ is Borel) and $\left(1^{\prime}\right)$ fails, then $G=G_{L}(A)$ is meager somewhere (i.e. $G \cap U$ is meager for some $U$ ).

Proof. From the hypothesis we have the dual assertion (4). Hence there exist $U_{0}^{*}$ and $k_{0}^{*}$ for which (5) holds (with $U_{0}^{*}$ for $U_{0}, k_{0}^{*}$ for $k_{0}$ ), and so also its equivalent $\left(5^{\prime}\right)$. Applying 5.1 to $\left(5^{\prime}\right)$ we see that the set

$$
\begin{aligned}
H & =\left\{x \in U_{0}^{*}: \forall k_{0}^{\prime} \exists k_{1}^{\prime} \cdots\left\{n: x \in B_{k_{0}^{\prime} \cdots k_{n}^{\prime}}\right\} \in \sim L\right\} \\
& =\left\{x \in U_{0}^{*}: \forall k_{1} \exists k_{2} \cdots\left\{n: A_{k_{0}^{*} k_{1} \cdots k_{n}}\right\} \notin L\right\}
\end{aligned}
$$


is comeager in $U_{0}^{*}$. But

$$
H^{\prime}=\left\{x \in U_{0}^{*}: \exists k_{0} \forall k_{1} \cdots\left\{n: x \in A_{k_{0} \cdots k_{n}}\right\} \notin L\right\} \supseteq H
$$

and $H^{\prime} \subseteq \sim G$ (as no game and its dual hold simultaneously). Hence $\sim G \cap U_{0}^{*}$ is comeager in $U_{0}^{*}$, so $G$ is meager somewhere, proving 5.3.

It might be remarked that the conclusion of 5.3 always holds if $X$ is not a Baire space.

We are now ready to prove $(A)$ and $(B)$ and even Kechris-style strengthenings $\left(A^{\prime}\right)$ and $\left(B^{\prime}\right)$ in which it is only assumed that $\left(1^{\prime}\right)$ is determinate. As noted in 2.1 , for $(A)$ and (B) we can safely assume all $A_{s}$ are open.

THEOREM ( $\left.\mathrm{A}^{\prime}\right)$. If $L$ is Borel (or, more generally, if $\left(\mathrm{1}^{\prime}\right)_{U}$ is determinate for each $U \in \Re)$, then $G=G_{L}(A)$ has the Baire property.

Proof. $G_{I}$ is a Boolean operation, and it follows (as is well known and easily seen) that $G_{L}^{U}\left(\left(A_{s} \cap U: s \in \operatorname{Sq}(\omega)\right)\right)=G_{I}^{X}(A) \cap U$. Hence by 5.1 and 5.3 (both applied with $U$ for $X$ ) we have

(6) For any open $U \neq \varnothing, G \cap U$ is comeager in $U$ or meager somewhere in $U$.

Hence

$\left(6^{\prime}\right)$ For any open $€ \neq \varnothing, G$ is comeager somewhere in $C^{\prime}$ or meager somewhere in e.

But (6') implies that $G$ has the Baire property (see $[6, \S 11$, IV]). Q.E.D.

The idea of using such a condition here to imply Baire property is again due to Kechris.

Before $\left(B^{\prime}\right)$ we draw another conclusion from the equivalence of $(5)$ and $\left(5^{\prime}\right)$ above, together with 5.1 .

5.4. Let $X$ be a Baire space. Then (1) and (1') are equivalent. Moreover (1) is determinate if and only if $\left(1^{\prime}\right)$ is.

Proof. As we observed in the proof of 5.1, if either (1) or $\left(1^{\prime}\right)$ holds then that game can be played so that in each play $\left(U_{0}, k_{0}, U_{1}, k_{1}, \ldots\right), A_{k_{0} \cdots k_{n}}$ is revolved by $U_{n+1}$. But for such resolved play, $\exists m U_{m} \subseteq A_{k_{0} \cdots k_{n}}$ is equivalent to $\exists m U_{m} \leqslant A_{k_{0} \cdots k_{n}}$. Indeed one direction is obvious, and if $\exists m U_{m} \leqslant A_{k_{0} \cdots k_{n}}$ but not $\exists m U_{m} \subseteq A_{k_{0} \cdots k_{n}}$, then $U_{n+1}$ is disjoint from $A_{k_{0} \ldots k_{n}}$, and it follows easily that $U_{n+1}$ is meager, which is impossible in a Baire space. Thus (1) and $\left(1^{\prime}\right)$ are equivalent.

If, say, $\left(1^{\prime}\right)$ is determinate and $\left(1^{\prime}\right)$ fails, then (5) holds. Hence for some $U_{0}$ and $k_{0}$, $\left(5^{\prime}\right)$ holds. By the part of 5.4 already proved (for $\left.X=U_{0}\right)\left(5^{\prime}\right)$ is equivalent to itself with ' $\leqslant$ ' replacing ' $\subseteq$ '. Hence, clearly, (1) holds, as desired.

It is clear (using the remarks at the beginning of the proof of (A) above) that Theorem $(\mathrm{B})$ reduces to the case when $\theta=X$. We state and prove $\left(\mathrm{B}^{\prime}\right)$ only for the case $\theta=X$.

TheOREM $\left(\mathrm{B}^{\prime}\right)$. Let $X$ be a Baire space. If $(1)$ is determinate (in particular if $L$ is Borel) then the condition ' $G$ is comeager' and (1) (and $\left.\left(1^{\prime}\right)\right)$ are equivalent.

Proof. By 5.4, (1) and $\left(1^{\prime}\right)$ are equivalent and determinate together. By 5.1, $\left(1^{\prime}\right)$ implies that $G$ is comeager. Assume (1) is determinate and $\left(1^{\prime}\right)$ fails. Then by 5.3, 
$G \cap U$ is meager for some $U$. Hence if $G$ were also comeager, $U$ itself would be meager, contradicting the fact that $X$ is a Baire space. This completes the proof.

We will now sharpen (1) in a way that will be especially useful in $§ 7$. We first need

Lemma 5.5. If $A$ and $B$ are open sets and $A \$ B$, then the set $A-B$ has nonempty interior.

Proof. Since $A \$ B$, by definition $A-B$ is not meager, and so, in particular, not nowhere dense. Thus there is a nonempty open $\Theta \subseteq \overline{A-B}$. But $\overline{A-B}=\bar{A}-B$ (since $\sim$ i is closed), and so $\Theta \cap A \subseteq A \cap(\bar{A}-B)=A-B$. Thus is suffices to

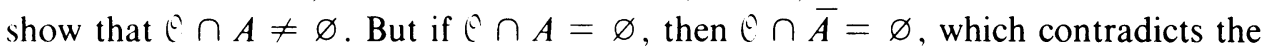
fact that $\cong \subseteq \bar{A}$. This completes the proof.

We will now show that $\left(l^{\prime \prime}\right)$, immediately below, is equivalent to (1). Observe that $\left(1^{\prime \prime}\right)$ is a "sharper" form of (1).

Proposition 5.6. Suppose that $X$ is a Baire space. Then if

$$
\forall U_{0} \forall k_{0} \exists U_{1} \subseteq U_{0} \exists k_{1} \cdots\left\{n: \exists m U_{m} \leqslant A_{k_{0} \cdots k_{n}}\right\} \in L,
$$

then

$$
\forall U_{0} \forall k_{0} \exists U_{1} \subseteq U_{0} \exists k_{1} \cdots\left\{n: U_{n} \leqslant A_{k_{0} \cdots k_{n}}\right\} \in L .
$$

Proof. First apply 4.3 to (1) with $(C, \leqslant)=(\mathscr{T}, \subseteq), a_{s}=A_{s}, K=\omega$, and $Q(U / E, k)$ if and only if $\left\{n: \exists m\left(U_{m} \leqslant A_{k_{0} \ldots k_{n}}\right)\right\} \in L$ and conclude that there is a strategy $G$ for (1) in which, for any play $\left(U_{0}, k_{0}, U_{1}, k_{1}, \ldots\right)$ of (1) using $G$, for every odd number $n, U_{n}$ resolves $A_{k_{0} \cdots k_{n}}$ (we do not need the rest of the conclusion of 4.3). As was remarked in the proof of 5.1, this says that for all odd $n$, either $U_{n} \subseteq A_{k_{0}} \cdots k_{n}$ or $U_{n} \cap A_{k_{0} \cdots k_{n}}=\varnothing$, and it follows easily that

(*) for all odd $n, \exists m U_{m} \leqslant A_{k_{0} \cdots k_{n}}$ if and only if $U_{n} \leqslant A_{k_{0} \cdots k_{n}}$.

We shall now modify the strategy $G$ so that $(*)$ holds for even $n$ as well. As play progresses, II will 'simulate' another play of game (1) going on at the same time in which he (II) is following the strategy $G$.

Say I plays $\left(U_{0}, k_{0}\right)$.

If $U_{0} \leqslant A_{k_{0}}$, II simulates that I has played $U_{0}^{\prime}=U_{0}$.

If $U_{0} \$ A_{k_{0}}$, by the lemma just proved, $U_{0}-A_{k_{0}}$ has nonempty interior. II simulates that I has played some $U_{0}^{\prime} \subseteq U_{0}-A_{k_{0}}$.

Then in both the simulated and the real games, II plays $\left(U_{1}, k_{1}\right)=G\left(\left(U_{0}^{\prime}, k_{0}\right)\right)$. Note that if $U_{0} \$ A_{k_{0}}$, then since $U_{1} \subseteq U_{0}^{\prime} \subseteq \sim A_{k_{0}}$, we have

$$
\text { if } U_{0} \$ A_{k_{0}} \text {, then } \forall V \in \mathscr{B}\left(V \subseteq U_{1} \rightarrow V \$ A_{k_{0}}\right) \text {. }
$$

Now say I plays $\left(U_{2}, k_{2}\right)$.

If $U_{2} \leqslant A_{k_{0} k_{1} k_{2}}$, II simulates $U_{2}^{\prime}=U_{2}$.

If $U_{2} \neq U_{2}-A_{k_{0} k_{1} k_{2}}$, again $U_{2}-A_{k_{0} k_{1} k_{2}}$ has nonempty interior, so II simulates $U_{2}^{\prime} \subseteq U_{2}-A_{k_{0} k_{1} k_{2}}$ and plays in both games $\left(U_{3}, k_{3}\right)=G\left(\left(U_{0}^{\prime}, k_{0}, U_{2}^{\prime}, k_{2}\right)\right)$. As before,

$\left(*_{2}\right) \quad$ if $U_{2} \$ A_{k_{0} k_{1} k_{2}}$, then $\forall V \in \Re\left(U \subseteq U_{3} \rightarrow V \$ A_{k_{0} k_{1} k_{2}}\right)$. 
Play continues in this manner, producing a simulated play $z^{\prime}=$ $\left(U_{0}^{\prime}, k_{0}, U_{1}, k_{1}, U_{2}^{\prime}, k_{2}, \ldots\right)$ played according to $G$, and an actual play $z=$ $\left(U_{0}, k_{0}, U_{1}, k_{1}, U_{2}, \ldots\right)$. Since $G$ was a winning strategy for (1), $Q\left(z^{\prime}\right)$ holds, which clearly implies $Q(z)$. Thus our strategy is a winning strategy for (1). We now show that for all $n, \exists m U_{m} \leqslant A_{k_{0} \cdots k_{n}}$ if and only if $U_{n} \leqslant A_{k_{0} \cdots k_{n}}$.

Indeed, for $n$ odd, this follows immediately from $(*)$. For $n$ even, right to left is trivial, so suppose $U_{n} \neq A_{k_{0} \cdots k_{n}}$. Then by $\left(*_{n}\right)$, if $v \in \mathscr{B}$ and $V \subseteq U_{n+1}$, then $V \$ A_{k_{0} \cdots k_{n}}$. This implies that, for all $m \geqslant n+1, U_{m} \$ A_{k_{0} \cdots k_{n}}$. For $m \leqslant n$, $U_{m} \$ A_{k_{0} \cdots k_{n}}$, since $U_{m} \supseteq U_{n}$. This establishes (**).

Thus since we have $\left\{n: \exists m U_{m} \leqslant A_{k_{0} \cdots k_{n}}\right\} \in L$, we have $\left\{n: U_{n} \leqslant A_{k_{0} \cdots k_{n}}\right\} \in L$ as well by $(* *)$. This completes the proof.

We remark that the analogue of 5.6 with $\left(1^{\prime}\right)$ in the place of (1) fails.

By exactly the same argument, using $4.3^{\prime}$ in place of 4.3 , we have

Proposition 5.6'. If $X$ is a Baire space, then if

$$
\exists U_{0} \exists k_{0} \forall U_{1} \subseteq U_{0} \forall k_{1} \cdots\left\{n: \exists m U_{m} \leqslant A_{k_{0} \cdots k_{n}}\right\} \notin L,
$$

then

$$
\exists U_{0} \exists k_{0} \forall U_{1} \subseteq U_{0} \forall k_{1} \cdots\left\{n: U_{n} \leqslant A_{k_{0} \cdots k_{n}}\right\} \notin L .
$$

This if game (1) is determinate, 5.6' provides the converse to 5.6. (In particular, if $L$ is Borel, (1) is a Borel game by an easier version of the argument for 5.2.) We therefore have

THEOREM ( $\left(\mathrm{B}^{\prime \prime}\right)$. If $L$ is Borel, then ( 1$)$ is equivalent to $\left(1^{\prime \prime}\right)$, and hence $\left(1^{\prime \prime}\right)$ is equivalent to the condition ' $G_{L}(A)$ is comeager'.

We close this section by discussing some alternative forms of the conditions $\left(1^{\prime}\right)$ and $\left(1^{\prime \prime}\right)$. As usual $[12, \S 21]$, if $I$ is the ideal of all meager sets, we can form the Boolean algebra $\mathscr{P}(X) / I$, and the important subalgebra $B P / I=\mathfrak{B}=\mathfrak{B}(X)$ (where $B P$ is the field of all sets having the Baire property). $B$ is complete (see $[12, \S 21])$. Its nonzero elements we denote by subscripted $b$. Let subscripted $U^{\prime}$ range over nonmeager open sets in $X$. It is easy to see that the following conditions $\left(1^{\prime \prime \prime}\right)$ and $\left(1^{\prime \prime \prime \prime}\right)$ are equivalent.

$$
\begin{gathered}
\forall b_{0} \forall k_{0} \exists b_{1} \leqslant b_{0} \exists k_{1} \cdots\left\{n: b_{n} \leqslant\left(A_{k_{0} \cdots k_{n}} / I\right)\right\} \in L . \\
\forall U_{0}^{\prime} \forall k_{0} \exists U_{1}^{\prime} \subseteq U_{0}^{\prime} \exists k_{1} \cdots\left\{n: U_{n}^{\prime} \leqslant A_{k_{0} \cdots k_{n}}\right\} \in L .
\end{gathered}
$$

(Suppose $\left(1^{\prime \prime \prime}\right)$ holds. In game $\left(1^{\prime \prime \prime \prime}\right)$ let I play $\left(U_{0}^{\prime}, k_{0}\right)$. Put $b_{0}=U_{0}^{\prime} / I$ and, by the strategy for $\left(1^{\prime \prime \prime}\right)$ applied to $\left(b_{0}, k_{0}\right)$, get $b_{1} \leqslant b_{0}$ and $k_{0}$. Choose $V$ so $V / I=b_{1}$, and let $U_{1}^{\prime}=U_{0}^{\prime} \cap V$. Then $U_{1}^{\prime} \subseteq U_{0}^{\prime}$ and $U_{1}^{\prime} / I=b_{1}$. Continuing, we clearly win in (1"'"'). The reverse argument is similar.)

Clearly if $X$ is a Baire space, $\left(1^{\prime \prime \prime \prime}\right)$ is identical with $\left(1^{\prime \prime}\right)$. All the same things hold for games starting with $\exists$, and hence $\left(1^{\prime \prime \prime}\right)$ and $\left(1^{\prime \prime \prime \prime}\right)$ are determinate together. Of course, if $L$ is Borel, the games $\left(1^{\prime \prime \prime}\right)$ and $\left(1^{\prime \prime \prime \prime}\right)$ are Borel. 
Now $[6, \S 10, \mathrm{~V}]$, in any space $X$, let $E$ be the union of all meager open sets, and let $D$ be $\sim E$; then $D$ is a Baire space. $D$ is comeager in $X$, and $D$ is regular closed ( $D=\bar{\Theta}$ for some open $\Theta$ ). It is easy to see that our $\mathfrak{B}(X)$ and $\mathscr{B}(D)$ are canonically isomorphic, and hence ( $\left(1^{\prime \prime \prime}\right)$ on $X$ and $\left(1^{\prime \prime \prime}\right)$ on $D$ coincide. We now see that $\left(1^{\prime \prime \prime}\right)$ (or $\left.\left(1^{\prime \prime \prime \prime}\right)\right)$ is the 'best' condition, since we have

( $\left.\mathrm{B}^{\prime \prime \prime}\right)$ For any space $X$, if $\left(1^{\prime \prime \prime}\right)$ is determinate (or if $L$ is Borel), then the condition ' $G$ is comeager' and $\left(1^{\prime \prime \prime}\right)$ are equivalent. Hence, if $L$ is Borel, then for any $b$,

$$
\forall b_{0} \leqslant b \forall k_{0} \exists b_{1} \leqslant b_{0} \exists k_{1} \cdots\left\{n: b_{n} \leqslant A_{k_{0} \cdots k_{n}} / I\right\} \in L
$$

if and only if $G / I \leqslant b$.

$\left(\mathrm{B}^{\prime \prime \prime \prime}\right)$ holds because both conditions are equivalent to their own versions on $D$, where $\left(1^{\prime \prime \prime}\right)$ is the same as $\left(1^{\prime \prime}\right)$, so equivalent to comeager by $\left(\mathrm{B}^{\prime \prime}\right)$.

Aside. It can be rather easily shown that if the now odd condition ( $\left(1^{\prime}\right)$ holds for a general $X$, then it holds for $D(X)$ (or indeed any regular closed subspace). Thus 5.1 would be no weaker if restricted to Baire spaces.

Let $L$ be Borel. The operation $G_{L}$ also produces an $\operatorname{Sq}(\omega)$-ary algebraic operation $\Gamma_{L}$ on each complete Boolean algebra $\mathfrak{B} . \mathfrak{B}$ can be represented (in many ways) as $\mathfrak{B}(X)$ (as above). Then we put

$$
\Gamma_{L}\left(\left(a_{s}: s \in \mathrm{Sq}(\omega)\right)\right)=G_{L}\left(\left(A_{s}: s \in \mathrm{Sq}(\omega)\right)\right) / I
$$

whenever $A_{s} / I=a_{s}$ (well defined for our $X$ by 2.1 and (A)). Now by the second part of $\left(\mathrm{B}^{\prime \prime \prime}\right)$, we see that $G / I$ comes out the same no matter which $X$ as above is taken. In other words $G / I$ can be defined within $B$ as the unique $c$ such that, for any $d \in \mathfrak{B}, d \leqslant c$ if and only if

$$
\forall b_{0} \leqslant d \forall k_{0} \cdots\left\{n: b_{n} \leqslant a_{k_{0} \cdots k_{n}}\right\} \in L
$$

6. Corresponding results for $\kappa$-meager. Let $\kappa$ be a fixed infinite cardinal, and let subscripted $\alpha, \beta, \gamma$ range over $\kappa . X$ is still any ordinary topological space. As promised in $\S 3$, we now give a new proof of the pivotal $(\mathrm{C})$ which applies also to the $\kappa$-notions.

THEOREM $(\mathrm{C})_{\kappa}$. If

$$
\forall U_{0} \forall \alpha_{0} \exists U_{1} \subseteq U_{0} \exists \alpha_{1} \cdots\left(\bigcap_{m \in \omega} U_{m} \subseteq\{x: P x \alpha\}\right),
$$

then $\left\{x: \forall \alpha_{0} \exists \alpha_{1} \cdots P x \alpha\right\}$ is $\kappa$-comeager. (Compare [9].)

Proof of $(\mathrm{C})_{\kappa}$. Let $F$ be a winning strategy as guaranteed by $(*)$. We will define an indexed family $\left(S_{s}: s \in \mathrm{Sq}(\kappa)\right)$ of sets $S_{s}$ of 'partial plays'. Here, by a partial $F$-play, or just a play for short, we mean a finite sequence $z=$ $\left(U_{0}, \alpha_{0}, V_{0}, \beta_{0}, \ldots, U_{n}, \alpha_{n}, V_{n}, \beta_{n}\right)$, where $U_{0} \supseteq V_{0} \supseteq U_{1} \supseteq \cdots \supseteq V_{n}$ and strategy $F$ has been followed. A play $z^{\prime}=\left(U_{0}^{\prime}, \alpha_{0}^{\prime}, V_{0}^{\prime}, \beta_{0}^{\prime}, \ldots, V_{n}^{\prime}, \beta_{n}^{\prime}\right)$ is called disjoint from $z$ if $V_{n} \cap V_{n}^{\prime}=\varnothing$.

For each $\alpha_{0}$ let $S_{\left(\alpha_{0}\right)}$ be a maximal pairwise disjoint set of plays $\left(U_{0}, \alpha_{0}, V_{0}, \beta_{0}\right)$. The set

$$
T_{\left(\alpha_{0}\right)}=\bigcup\left\{V_{0}: \text { for some } U_{0}, \beta_{0},\left(U_{0}, \alpha_{0}, V_{0}, \beta_{0}\right) \in S_{\left(\alpha_{0}\right)}\right\}
$$


is open and dense. Indeed, if for some $U, U \subseteq \sim T_{\left(\alpha_{0}\right)}$, then the (unique) play $\left(U, \alpha_{0}, V, \beta_{0}\right)$ could be added to $S_{\left(\alpha_{0}\right)}$, contrary to its maximality.

For every $\left(\alpha_{0}, \alpha_{1}\right)$, let $S_{\left(\alpha_{0}, \alpha_{1}\right)}$ be a maximal pairwise disjoint set of plays $\left(U_{0}, \alpha_{0}, V_{0}, \beta_{0}, U_{1}, \alpha_{1}, V_{1}, \beta_{1}\right)$ such that $\left(U_{0}, \alpha_{0}, V_{0}, \beta_{0}\right) \in S_{\left(\alpha_{0}\right)}$. As before, it turns out that

$$
T_{\left(\alpha_{0}, \beta_{0}\right)}=\bigcup\left\{V_{1}: \text { for some } U_{0}, \beta_{0}, U_{1}, \beta_{1},\left(U_{0}, \alpha_{0}, \ldots, V_{1}, \beta_{1}\right) \in S_{\left(\alpha_{0}, \alpha_{1}\right)}\right\}
$$

is open and dense. Indeed, suppose some $U \subseteq \sim T_{\left(\alpha_{0}, \alpha_{1}\right)}$. Since $T_{\left(\alpha_{0}\right)}$ is dense and open, $U$ intersects it, and hence for some $\left(U_{0}, \alpha_{0}, V_{0}, \beta_{0}\right) \in S_{\left(\alpha_{0}\right)}$, the open set $W=U \cap V_{0} \neq \varnothing$. Then the play $\left(U_{0}, \alpha_{0}, V_{0}, \beta_{0}, W, \alpha_{1}, V, \beta_{1}\right)$ could be added to $S_{\left(\alpha_{0}, \alpha_{1}\right)}$, contradicting maximality as before.

Continuing inductively in this way, $S_{\left(\alpha_{0} \ldots \alpha_{n}\right)}$ and $T_{\left(\alpha_{0} \ldots \ldots \alpha_{n}\right)}$ are defined in general. Since the $(\kappa)$ sets $S_{s}, s \in \operatorname{Sq}(\kappa)$, are all dense open, the set $H=\cap\left\{T_{s}: s \in \operatorname{Sq}(\kappa)\right\}$ is $\kappa$-comeager. Hence it suffices to show that for all $x \in H$,

$$
\forall \alpha_{0} \exists \beta_{0} \forall \alpha_{1} \exists \beta_{1} \cdots P\left(x,\left(\alpha_{0}, \beta_{0}, \alpha_{1}, \ldots\right)\right) .
$$

Suppose $x \in H$. We give II's winning strategy for $(* *)$.

Suppose I plays $\alpha_{0}$. Now $x \in T_{\left(\alpha_{0}\right)}$, and since $S_{\left(\alpha_{0}\right)}$ is pairwise disjoint,

$$
\text { there is exactly one }\left(U_{0}, \alpha_{0}, V_{0}, \beta_{0}\right) \in S_{\left(\alpha_{0}\right)} \text { such that } x \in V_{0} \text {. }
$$

In (**), let II play $\beta_{0}$. Suppose next I plays $\alpha_{1}$. Again, since $x \in T_{\left(\alpha_{0}, \alpha_{1}\right)}$,

(8) there is exactly one $\left(U_{0}^{\prime}, \alpha_{0}, V_{0}^{\prime}, \beta_{0}^{\prime}, U_{1}, \alpha_{1}, V_{1}, \beta_{1}\right) \in S_{\left(\alpha_{0}, \alpha_{1}\right)}$ such that $x \in V_{1}$.

Now $x \in V_{1}$ and so $x \in V_{0}$, and $\left(U_{0}^{\prime}, \alpha_{0}, V_{0}^{\prime}, \beta_{0}^{\prime}\right) \in S_{\left(\alpha_{0}\right)}$, by definition, so by (7), $U_{0}^{\prime}$, $V_{0}^{\prime}$, and $\beta_{0}^{\prime}$ are $U_{0}, V_{0}$, and $\beta_{0}$. II plays $\beta_{1}$ in game $(* *)$.

II plays in a similar way throughout the game.

Clearly, if $\left(\alpha_{0}, \beta_{0}, \alpha_{1}, \beta_{1}, \ldots\right)$ is a full play of $(* *)$ by the above strategy, then there is a (unique)

$$
\left(U_{0}, \alpha_{0}, V_{0}, \beta_{0}, U_{1}, \alpha_{1}, \ldots\right)
$$

such that $x \in V_{n}$, and for each $n,\left(U_{0}, \alpha_{0}, \ldots, V_{n}, \beta_{n}\right) \in S_{\left(\alpha_{0}, \ldots, \alpha_{n}\right)}$. From the definition of the $S_{s}$, we infer that (9) is a play of the game (*) by the winning strategy $F$. Hence $\bigcap_{n \in \omega} V_{n} \subseteq\left\{x: P\left(x,\left(\alpha_{0}, \beta_{0}, \ldots\right)\right)\right\}$. Since $x \in \cap_{n \in \omega} V_{n}$, we have $P\left(x,\left(\alpha_{0}, \beta_{0}, \ldots\right)\right)$. Thus our strategy for $(* *)$ has won, completing the proof.

Now that we have a proof of $(C)_{\kappa}$, the reader may verify that the remainder of $\$ 5$ from 5.1 to $\left(\mathrm{B}^{\prime \prime \prime}\right)$ can be read correctly verbatim in the $\kappa$-version, that is, substituting the notion $\kappa$-meager, $\kappa$-Baire property, $\leqslant_{\kappa}, \equiv_{\kappa}, \kappa$-Baire space for their respective ordinary versions, and taking $K=\kappa$, that is, letting subscripted $k$ range over $\kappa$ in all games $\forall k_{0} \exists k_{1} \cdots$ and $\forall U_{0} \forall k_{0} \exists U_{1} \exists k_{1} \cdots$. (Note that $L$ is still a subset of $\mathscr{P}(\omega)$, and when $L$ is Borel in $\S 5$, it is still ordinary Borel.)

These remarks must be clarified in connection with reference to [6] concerning the discussion just before $\left(\mathrm{B}^{\prime \prime \prime}\right)$ of the sets $D$ and $E$. It is known (but it is not so easy to give a reference) that all these things hold in the $\kappa$-version. In fact, if one starts at [6, $\S 10]$ (sets of the first category), the entire discussion, at least to just before Corollary $1, \S \S I I, I V-e n o u g h$ for us-holds in the $\kappa$-version essentially verbatim. The only case where this is not pretty clear in advance is the critical Banach Theorem on 
Additivity ( $\$ 10$; III), now read: the union of $\kappa$-meager sets each open in their union is $\kappa$-meager. But here also the proof in [6] works perfectly for $\kappa$. So $\$ 5$ (in the $\kappa$-version) shows

THEOREM 6.1. Everything in $\$ 5$ holds in the $\kappa$-version. In particular, if $L$ is Borel then

(A) $)_{\kappa}$ The Borel $\kappa$-game operation $G_{L}^{X, \kappa}$ preserves the $\kappa$-Baire property.

(B) ${ }_{\kappa}$ If $X$ is a $\kappa$-Baire space and the sets $A_{s}, s \in \mathrm{Sq}(\kappa)$, have the $\kappa$-Baire property, then $G_{L}^{X, \kappa}(A) \leqslant_{\kappa} U$ if and only if

$$
\forall U_{0} \forall \alpha_{0} \exists U_{1} \subseteq U_{0} \exists \alpha_{1} \cdots\left\{n: \exists m U_{m} \leqslant{ }_{\kappa} A_{\alpha_{0} \cdots \alpha_{n}}\right\} \in L .
$$

$\left(\mathrm{B}^{\prime \prime}\right)_{\kappa}$ Under the hypotheses of $(\mathrm{B})_{\kappa}$, the equivalent conditions in the conclusion of $(\mathrm{B})_{\kappa}$ are also equivalent to

$$
\forall U_{0} \forall \alpha_{0} \exists U_{1} \subseteq U_{0} \exists \alpha_{1} \cdots\left\{n: U_{n} \leqslant{ }_{\kappa} A_{\alpha_{0} \cdots \alpha_{n}}\right\} \in L .
$$

By analogy with the $C$-sets (see, e.g., [13]), let $C_{L}^{X, \kappa}$ be the least class of subsets of $X$ containing the open sets, and closed under complement, $\leqslant \kappa$-union, and $G_{L}^{X . \kappa}$. From $(A)_{\kappa}$ we obtain at once

COROLlaRY 6.2. If $L$ is Borel, then all $C_{L}^{X, \kappa}$-sets have the $\kappa$-Baire property.

7. Action and logic. In this section we discuss applications of Theorem $(B)_{\kappa}$ in connection with group actions (in particular the logic action) and the *-transform of [13]. This discussion will be given at once for the general $\kappa$-case, as the case $\kappa=\omega$ has essentially been dealt with by Burgess [1].

Let $G$ be a topological group which is a $\kappa$-Baire space and which acts continuously on a topological space $X$. (The 'product case' of [13] and the case where $X$ is a Borel space (see Miller [8]) could also be considered.) In this section, $G$ plays the role played by $X$ in the rest of this paper; $\mathscr{B}$ is a weak basis for $G$, and subscripted $U$ will range over $\mathscr{B}$.

If $B \subseteq X$ and $x \in X$, put $B^{x}=\{g \in G: g x \in B\}$. If $\theta \subseteq G$ is nonempty and open, put $B^{* \theta}=\left\{x \in X: B^{x} \leqslant_{\kappa} \vartheta\right\} . B^{*}=B^{* G} . A \subseteq X$ is called $\kappa$-normal if $A^{x}$ has the $\kappa$-Baire property for all $x \in X$.

The behavior of the *-transform on closed sets and over complements, intersections of length at most $\kappa$, and the operation (A) is discussed in [13]. In particular, we know that $B^{*}$ is invariant (under the group action), and that $B=B^{*}$ if and only if $B$ is invariant. Also

$$
\begin{aligned}
& \text { if } B \text { is closed, so is } B^{*} \text {, } \\
& \left(\bigcap_{\alpha<\kappa} B_{\alpha}\right)^{*}=\bigcap_{\alpha<\kappa} B_{\alpha}^{*} .
\end{aligned}
$$

$$
\text { If } B \text { is } \kappa \text {-normal, then }(\sim B)^{*}=\sim \bigcup_{U \in G B} B^{* U} \text {. }
$$

It follows that if $G$ has a weak basis of cardinality $\leqslant \kappa$, and $B$ is $\kappa$-Borel, then so is $B^{*}$. The following theorem (a corollary of $\left.\left(\mathrm{B}^{\prime \prime}\right)_{\kappa}\right)$ shows how ${ }^{*}$ behaves with respect to the operation $G_{L}^{X, \kappa}$. 
Theorem 7.1. Suppose L is Borel. Then

(a) All $G_{L}^{X, \kappa}$-sets are $\kappa$-normal.

(b) Let $A_{s} \subseteq X$ be $\kappa$-normal for all $s \in \mathrm{Sq}(\kappa)$ and let $H=G_{L}^{X, \kappa}(A)$. Then if $(\hat{\subseteq} \subseteq G$ is open and nonempty and $x \in X$, then $x \in H^{*}$ if and only if

$$
\forall U_{0} \subseteq \vartheta \forall \alpha_{0} \exists U_{1} \subseteq U_{0} \exists \alpha_{1} \cdots\left\{n: x \in A_{\alpha_{0}{ }^{\prime} \cdots \alpha_{n}}^{* U_{n}}\right\} \in L
$$

Proof. (a) follows immediately from 6.2 since the inverse image $B^{x}$ of a $C_{L}^{X .{ }^{\kappa}}$-set is clearly $C_{L}^{G, \kappa}$.

By definition, $x \in A^{* U_{n}}$ if and only if $U_{n} \leqslant{ }_{\kappa} A_{s}^{x}$. Hence (b) is an immediate consequence of $\left(\mathrm{B}^{\prime \prime}\right)_{\kappa}$.

For $1 \leqslant \xi<\omega_{1}$, let $C_{\xi}^{x, \kappa}$ be the least class of subsets of $X$ containing the open sets, and closed under complement, union of length at most $\kappa$, and the operations $G_{L}^{x, \kappa}$, where $L$ ranges over all $\Pi_{\xi}^{0}$ subsets of $\mathscr{P}(\omega)$. (See $\S 2$.)

COROLlaRY 7.2. If $G$ has a weak basis of cardinality at most $\kappa$, and $B$ is a $C_{\xi}^{X, \kappa}$ set, then so is $B^{*}$.

Proof. The proof is by induction on the formation of the $C_{\xi}^{X, \kappa}$ set $B$. The cases when $B$ is closed or the $\kappa$-union or complement of previous $C_{\xi}^{X, \kappa}$ sets follow immediately from (10)-(12) above (but note that the complement case uses 7.1(a), as does the case below).

Suppose that $B=G_{L}^{X, \kappa}(A)$, where $L$ is a $\Pi_{\xi}^{0}$ set, and each $A_{s}$ is a $C_{\xi}^{X, \kappa}$ set. To complete the proof, it clearly suffices to show that the game in 7.1(b) is equivalent to a game

$$
\forall U_{0} \forall \alpha_{0} \exists U_{1} \exists \alpha_{1} \cdots\left\{n: x \in A^{\prime}\left(U_{0}, \alpha_{0}, \ldots, U_{n}, \alpha_{n}\right)\right\} \in L^{\prime}
$$

where each set $A^{\prime}$ is an $A_{\alpha_{0}}^{* U_{n} \alpha_{n}}, X$, or $\varnothing$. and $L^{\prime}$ is a $\Pi_{\xi}^{0}$ subset of $\mathcal{P}(\omega)$.

Indeed, if $\xi=1$ (i.e., if $L$ is closed), this may be done almost exactly as in [13, Corollary 1.8]. For $\xi \geqslant 2$, the reader may check, using $\left(2^{\prime}\right)$ of $\S 2$, that if we set $U_{-1}=(1)$

$$
A^{\prime}\left(U_{0}, \alpha_{0}, \ldots, U_{n}, \alpha_{n}\right)= \begin{cases}X & \text { if } n=2 k+1, \text { and } U_{k} \subseteq U_{k-1}, \\ \varnothing & \text { if } n=2 k+1, \text { and } U_{k} \nsubseteq U_{k-1}, \\ A_{\alpha_{0} \cdots \alpha_{k}}^{* U_{k}} & \text { if } n=2 k,\end{cases}
$$

and $L^{\prime}=\{S \subseteq \omega:$ if for all even $k, 2 k+1 \in S$, then for all odd $k, 2 k+1 \in S$ and $\{k: 2 k \in S\} \in L\}$, this works.

Burgess [1] deduced 7.1 and 7.2 from Theorem (B) for the case $\kappa=\omega$ and $G$ Polish. For $\kappa=\omega$, our 7.1 and 7.2 have advanced from [1] only by replacing the hypothesis ' $G$ is Polish' by ' $G$ has a countable weak basis'. However for $\kappa$ uncountable, 7.1 and 7.2 are new.

Following $\$ 4$ of [13], we now apply 7.1 to an enriched version of the language $\varrho_{\kappa}{ }^{+} \kappa$. Henceforth we assume that $\kappa$ is regular and $2^{\lambda} \leqslant \kappa$ whenever $\lambda<\kappa$.

A similarity type of structures is a pair $\rho=\left(\rho^{\prime}, J\right)$ where $\rho^{\prime}: I \rightarrow \kappa .(M, S)$ is a $\rho$-structure if $S \in X_{\rho, M}=\prod_{i \in I} 2^{M^{\rho_{i}}} \times M^{J}$. (Thus $\alpha$-ary relations are allowed for $\alpha<\kappa$.) For simplicity assume $I \cup J$ has cardinality at most $\kappa$. (For a way of 
avoiding this assumption, see [13].) Set $X=X_{\rho}=X_{\rho, \kappa}$ with the $\kappa$-topology, which has as a basis all intersections of fewer than $\kappa$ sets which are open in the product topology. Let $G$ be the group $\kappa$ ! of all permutations of $\kappa$, also with the $\kappa$-topology. $G$ is a $\kappa$-Baire space (see [10]). $G$ has the basis $\mathscr{B}=\left\{[\sigma]: \sigma \in \kappa^{\kappa}\right.$, $\sigma$ nonrepeating $\}$, where $[\sigma]=\left\{g \in G: g^{-1} \supset \sigma\right\}$. (Henceforth we will use the letter $s$ to range over $\kappa^{\kappa}$ and $\sigma$ to range over nonrepeating elements of $\kappa^{\kappa}$. As usual, for any set $S, S^{\kappa}=$ $\cup_{\gamma<\kappa} S^{\gamma}$.) Note that, under our assumptions on $\kappa, \Re$ has cardinality $\kappa$.

Also, $G$ is a topological group which acts continuously on $X, g S$ being the usual isomorph of $S$ under $g$.

Let $\Pi_{\xi}^{0}, 1 \leqslant \xi<\omega_{1}$, be the usual hierarchy of Borel sets as in $\S 2$. For all $\xi$, $1 \leqslant \xi<\omega_{1}$, we will define a language $\mathcal{E}_{\kappa}{ }^{+}{ }_{\kappa} \xi$, which will be $\mathcal{L}_{\kappa}{ }^{{ }}{ }_{\kappa}$ with the addition of new operators which formalize the $\Pi_{\xi}^{0}$-game operations.

The language $\mathcal{L}_{\kappa^{+}{ }_{\kappa} G} \xi$ has $\rho^{\prime}(i)$-ary relation symbols $R_{i}(i \in I)$, individual constant symbols $c_{j}(j \in J)$, variables $u_{\eta}(\eta<\kappa), \approx, \sim, \forall, \wedge$, and the variable-binding operations $G^{L}$ (for all $\Pi_{\xi}^{0}$ subsets $L$ of $\mathcal{P}(\omega)$ ). Atomic formulas are as usual. The class of formulas is the smallest class containing the atomic formulas, and closed under $\sim, \forall W$ (where $W$ is a set of fewer than $\kappa$ variables), $\wedge S$ (where $S$ is a set of at most $\kappa$ formulas having fewer than $\kappa$ free variables), and under $G^{l}$. That is, suppose

$$
\Phi=\left(\varphi_{\alpha_{0} \ldots, \alpha_{n}}^{\beta_{0} \ldots, \beta_{n}}: n \in \omega, \alpha_{0}, \ldots, \alpha_{n}, \beta_{0}, \ldots, \beta_{n}<\kappa\right)
$$

is a system of formulas, and $W=\left(w_{\gamma}^{i}: i \in \omega, \gamma<\kappa\right)$ is a system of distinct variables. Further suppose that $V=\left\{v_{\eta}: \eta<\delta\right\}$ is a set of fewer than $\kappa$ variables, disjoint from $W$, and that the free variables of $\varphi_{\alpha_{0}, \ldots, \alpha_{n}}^{\beta_{0}, \ldots, \beta_{n}}$ are among $V \cup\left\{w_{\gamma}^{i}: i \leqslant n\right.$, $\left.\gamma<\beta_{i}\right\}$. Then $G_{W}^{L}(\Phi)$ is satisfied by a structure $(M, S)$ under the assignment $v_{\eta} \rightarrow m_{\eta}$ just in case

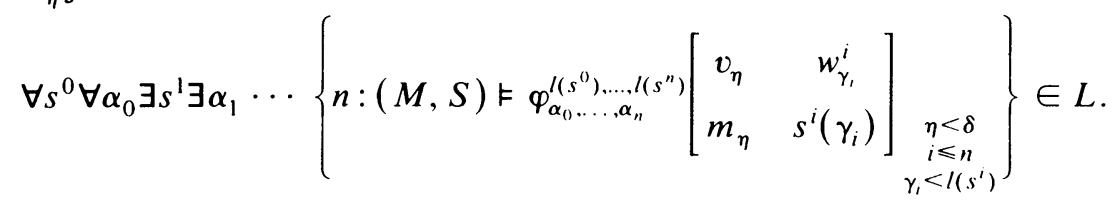

(Unlike the case $\kappa=\omega$ in [13] and [1], for $\kappa>\omega$ there is no equivalent language in which the game players play only one element of the model at a time, rather than a sequence of length $<\kappa$.)

From now on we consider only $\rho$-structures $(M, S)$ with $M=\kappa$, so $(M, S)$ can be identified with the point $S \in X$. A set $B \subseteq X$ is called an $\varrho_{\kappa^{+}{ }_{\kappa} G} \xi$-set if it is the class of models (with universe $\kappa$ ) of a sentence of $\mathcal{L}_{\kappa^{+}{ }_{\kappa G}} \xi$.

TheOREM 7.3 (BURGeSS FOR $\kappa=\omega$ ). Invariant $C_{\xi}^{X, \kappa}=\varrho_{\kappa^{+}{ }_{\kappa} G} \xi$ (that is, $B \subseteq X$ is an invariant $C_{\xi}^{X, \kappa}$-set if and only if $B$ is an $\mathcal{L}_{\kappa}{ }_{\kappa}{ }_{\kappa} \xi-$ set).

Proof. 7.3 is really a corollary of 7.1(b) by the same method as [13] and [1], so we sketch only the argument. The right-to-left direction is easy but heavily uses the hypothesis that $2^{\lambda} \leqslant \kappa$ for $\lambda<\kappa$. For the other direction, for $A \subseteq X$ and $\beta<\kappa$, let $A^{(* \beta)}=\left\{(S, \sigma) \in X \times \kappa^{\beta}: S \in A^{*[\sigma]}\right\}$. We will show

$$
\text { If } A \text { is a } C_{\xi}^{X, \kappa} \text {-set, and } \beta<\kappa \text {, then } A^{(* \beta)} \text { is an } \mathcal{L}_{\kappa^{+}{ }_{\kappa} G} \xi \text {-set (in } X \times \kappa^{\iota \beta} \text { ). }
$$


This will complete the proof, for if $A$ is also invariant, we will have shown that $A=A^{*}=A^{(* 0)}$ is an $\mathfrak{L}_{\kappa^{+}{ }_{\kappa} G} \xi$-set.

The cases when $A$ is open, or the complement of $\leqslant \kappa$-union of previous $C_{\xi}^{X, \kappa}$-sets are dealt with in [13]. Thus it will suffice to prove the following.

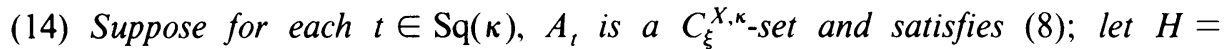
$G_{I}^{X, \kappa}\left(\left(A_{t}: t \in \operatorname{Sq}(\kappa)\right)\right)$, where $L$ is $\Pi_{\xi}^{0}$ in $\mathscr{P}(\omega)$. Then (13) holds for $H$.

Indeed, by hypothesis, for each $t \in \operatorname{Sq}(\kappa)$ and $\beta<\kappa$, there exists a formula $\varphi_{t}^{\beta}$ of $E_{\kappa}{ }^{\prime}{ }_{\kappa} \xi$ with free variables among $\left\{u_{\eta}: \eta<\beta\right\}$ such that, for all $S \in X$ and all nonrepeating $\sigma \in \kappa^{\beta}$,

$$
(S, \sigma) \in A_{t}^{(* \beta)} \text { if and only if }(\kappa, S) \vDash \varphi_{t}^{\beta}\left[\begin{array}{c}
u_{\eta} \\
\sigma(\eta)
\end{array}\right]_{\eta<\beta} .
$$

Applying 7.1 to the basis 6 , we see that for all such $(S, \sigma)$, the following are equivalent.

$$
\begin{gathered}
(S, \sigma) \in H^{(* \beta)}, \quad S \in H^{*}[\sigma], \\
\forall \sigma^{0} \supseteq \sigma \forall \alpha_{0} \exists \sigma^{1} \supseteq \sigma^{0} \exists \alpha_{1} \cdots\left\{n: S \in A_{\alpha_{0} \cdots \alpha_{n}}^{*\left[\sigma^{n}\right]}\right\} \in L . \\
\forall \sigma^{0} \supseteq \sigma \forall \alpha_{0} \exists \sigma^{1} \supseteq \sigma^{0} \exists \alpha_{1} \cdots\left\{n:\left(S, \sigma^{n}\right) \in A_{\alpha_{0} \cdots \alpha_{n}}^{*\left(l\left(\sigma^{n}\right)\right)}\right\} \in L . \\
\forall \sigma^{0} \supseteq \sigma \forall \alpha_{0} \exists \sigma^{1} \supseteq \sigma^{0} \exists \alpha_{1} \cdots\left\{n:(\kappa, S) \vDash \varphi_{\alpha_{0} \cdots \alpha_{n}}^{l\left(\sigma^{n}\right)}\left[\begin{array}{c}
u_{\eta} \\
\sigma^{n}(\eta)
\end{array}\right]{ }_{\eta<l\left(\sigma^{n}\right)}\right\} \in L .
\end{gathered}
$$

We must show that (16) is equivalent to an expression of the form $(*)$. We will use a coding like the one in the proof of 7.2. As in the case of 7.2, the case $\xi=1$ was essentially dealt with in [13], so we consider only $\xi \geqslant 2$.

Suppose that $\beta+\beta_{0}+\cdots+\beta_{n}=\delta$, and let $\left\{v_{\eta}: \eta<\beta\right\} \cup\left\{w_{\gamma_{i}}^{i}: \gamma_{i}<\beta_{i}, i \leqslant n\right\}$ be a set of distinct variables not occurring bound in $\varphi_{t}^{\delta}$. Recall that $\varphi_{t}^{\delta}$ has its free variables among $\left\{u_{\eta}: \eta<\delta\right\}$. Let $\hat{\varphi}_{t}^{\beta_{0} \cdots \beta_{n}}$ be the formula $\varphi_{t}^{\delta}$ but with the free occurrences of its first $\beta$ free variables $\left\{u_{\eta}: \eta<\beta\right\}$ replaced by $\left\{v_{\eta}: \eta<\beta\right\}$, its next $\beta_{0}$ free variables $\left\{u_{\eta}: \beta \leqslant \eta<\beta+\beta_{0}\right\}$ replaced by $\left\{w_{\gamma_{0}}^{0}: \gamma_{0}<\beta_{0}\right\}$, and so on.

Note that the assertion "s is nonrepeating" is expressible in our language. Let $\iota\left(\beta, \beta_{0}, \ldots, \beta_{n}\right)$ be the formula which says: "The variables $\left\{v_{\eta}: \eta<\beta\right\} \cup\left\{w_{\gamma_{i}}^{i}: \gamma_{i}<\right.$ $\left.\beta_{i}, i \leqslant n\right\}$ denote distinct objects." The reader will verify that $(* *)$ is equivalent to (16).

$(* *) \quad \forall s^{0} \forall \alpha_{0} \exists s^{1} \exists \alpha^{1} \cdots\left\{n:(\kappa, S) \vDash \psi_{\alpha_{0} \cdots \alpha_{n}}^{l\left(s^{0}\right) \cdots /\left(s^{\prime \prime}\right)}\left[\begin{array}{cc}v_{\eta} & w_{\gamma_{i}}^{i} \\ \sigma(\eta) & s^{i}\left(\gamma_{i}\right)\end{array}\right] \underset{\substack{\eta<\beta \\ i \leqslant n \\ \gamma_{i}<l\left(s^{i}\right)}}{\sigma}\right\} \in L^{\prime}$

where

$$
\psi_{\alpha_{0} \cdots \alpha_{n}}^{\beta_{0} \cdots \beta_{n}}=\left\{\begin{array}{l}
\iota\left(\beta, \beta_{0}, \ldots, \beta_{k-1}\right) \rightarrow \iota\left(\beta, \beta_{0}, \ldots, \beta_{k}\right) \text { if } n=2 k+1, \\
\hat{\varphi}_{\alpha_{0} \cdots \alpha_{k}}^{\beta_{0} \cdots \beta_{k}} \text { if } n=2 k,
\end{array}\right.
$$

and $L^{\prime}$ is as in the proof of 7.2. Note that $(* *)$ is in the desired form, completing the proof. 


\section{REFERENCES}

1. J. Burgess, Infinitary languages and descriptive set theory, Ph.D. thesis, Univ. of California, Berkeley, Calif., 1974.

2. J. E. Fenstad and D. Normann, On absolutely measurable sets, Fund. Math. 81 (1974), 91-98.

3. D. Gale and F. Stewart, Infinite games with perfect information. Contributions to the Theory of Games, Vol. II, Ann. of Math. Studies, no. 28, Princeton Univ. Press, Princeton, N. J., 1953, pp. 254-266.

4. A. Kechris, Measure and category in effective descriptive set theory, Ann. Math. Logic 5 (1973). $337-384$.

5. __ Forcing in analysis, Higher Set Theory (Proc. Conf. Oberwolfach, 1977), Lecture Notes in Math., vol. 669, Springer, Berlin, 1978, pp. 277-302.

6. K. Kuratowski, Topologv, Vol. I, Academic Press, New York; PWN, Warsaw, 1966.

7. D. A. Martin, Borel determinacy, Ann. of Math. (2) 102 (1975), 363-371.

8. D. Miller, Invariant descriptive set theory and the topological approach to model theory. Ph.D. thesis, Univ. of California, Berkeley, Calif., 1976.

9. J. C. Oxtoby, The Banach-Mazur game and Banach category theorem, Contributions to the Theory of Games, Vol. III, Ann. of Math. Studies, no. 28, Princeton Univ. Press, Princeton. N. J., 1957, pp. 159-163.

10. G. Reyes, Typical and generic relations in a Baire space for models, Ph.D. thesis, Univ. of California. Berkeley, Calif., 1967.

11. K. Schilling, Absolutely $\Delta_{2}^{\prime}$ operations and the Baire property, Abstracts Amer. Math. Soc. 1 (1980), 239. Abstract \#80T-E22.

12. R. Sikorski, Boolean algehras, 3rd ed., Ergeb. Math. Grenzgeb., Bd. 25, Springer, New York, 1969.

13. R. Vaught, Invariant sets in topology and logic, Fund. Math. 82 (1974), 269-294.

14. R. Vaught and K. Schilling, Borel game operations and the Baire property, Notices Amer. Math. Soc. 26 (1979), A-247. Abstract \#764-E1.

Graduate School of Management, University of California, Los Angeles, California 90024

Department of Mathematics, University of CALIfornia, Berkeley, California 94720 